\title{
Coeficiente del cultivo (Kc) del arroz a partir de lisímetro de drenaje en La Molina, Lima-Perú
}

\author{
Coefficient of cropping $(\mathrm{Kc})$ of rice from drain lysimeter in The Molina, Lima-Perú \\ Elfer Neira Huamán ${ }^{1}$, Lía Ramos Fernández ${ }^{1 *}$, Luis Ramón Razuri Ramírez ${ }^{1}$
}

\begin{abstract}
RESUMEN
Esta investigación se realizó en el Área Experimental de Riego (AER) de La Molina, Perú, a una altitud de 243,7 m.s.n.m. Se estimó la evapotranspiración del cultivo (ETc) de arroz variedad IR 71706 a partir de información obtenida de un lisímetro de drenaje y de la evapotranspiración de referencia (ETo) por medio del método FAO Penman-Monteith con datos de una estación meteorológica automática Davis, en el período del cultivo de diciembre del 2017 a abril del 2018. Se registraron lecturas diarias del riego, variación de la humedad del suelo y precipitación para realizar el balance de humedad del suelo y determinar la ETc y coeficiente del cultivo (Kc) durante el ciclo del cultivo. Se obtuvo un ETc máximo de $9.19 \mathrm{~mm} \mathrm{~d}^{-1}$ en la fase reproductiva (estado de huso y floración), un valor mínimo de $1,35 \mathrm{~mm} \mathrm{~d}^{-1}$ en la etapa final del cultivo, valor promedio de 5,02 $\mathrm{mm} \mathrm{d}^{-1}$, y ETc total de $658,23 \mathrm{~mm}$ con 587 macollos $\mathrm{m}^{-2}$ y con altura promedio de $0,80 \mathrm{~m}$. El Kc inicial, el medio y final variaron alrededor de 0,9; 1,70 y 0,84 , respectivamente.
\end{abstract}

Palabras clave: arroz, evapotranspiración del cultivo, evapotranspiración de referencia, coeficiente del cultivo, balance de humedad del suelo.

\begin{abstract}
This investigation was conducted at the Experimental Irrigation Area (AER, Spanish acronym) of La Molina, Peru, at an altitude of 243.7 m.a.s.l. The crop evapotranspiration (CET) was estimated for the IR 71706 rice variety using information obtained from a drainage lysimeter, and the reference evapotranspiration was calculated using the FAO Penman-Monteith method using information obtained from a Davis automatic weather station during the cultivation period from December 2017 to April 2018. Daily readings of irrigation, ground moisture variation, and precipitation were recorded to achieve soil moisture balance and thus determine the CET and crop coefficient $(K c)$ during the cultivation cycle. A maximum CET of $9.19 \mathrm{~mm} \mathrm{~d}^{-1}$ was obtained during the reproductive phase (spindle and flow state), a minimum CET of $1.35 \mathrm{~mm} \mathrm{~d}^{-1}$ during the final crop stage, and an average value of $5.02 \mathrm{~mm} \mathrm{~d}^{-1}$, and total CET of $658.23 \mathrm{~mm}$ with 587 clusters $\mathrm{m}^{-2}$, with an average height of $0.80 \mathrm{~m}$. The initial, mean, and final Kc were 0.9, 1.70, and 0.84, respectively.
\end{abstract}

Keywords: rice, crop evapotranspiration, reference evapotranspiration, crop coefficient, soil moisture balance.

\section{Introducción}

La FAO ubica al Perú en el puesto 17 del ránking de los países del mundo con mayor disponibilidad de agua. El $86 \%$ del agua dulce se utiliza para satisfacer la demanda agrícola (Tabra, 2013). Existen problemas con respecto a la dotación y aplicación del riego, en gran medida, por el desconocimiento del coeficiente del cultivo (Kc) y eficiencia de aplicación del riego para condiciones locales. Dentro del sistema agua-suelo-planta, parte del agua aportada, ya sea por precipitación o por riego, se pierde por percolación profunda, escorrentía superficial y por evapotranspiración. Lla evapotranspiración es la pérdida de agua debido a la evaporación directa de la superficie del suelo y la transpiración como respuesta de las necesidades hídricas de la planta para su crecimiento y desarrollo (Allen et al., 2006). Un método directo para la evaluación del balance hídrico en el sistema agua-suelo-planta es aislar una parte del sistema mediante lisímetros para medir el ingreso y salida de agua.

\footnotetext{
1 Departamento de Recursos Hídricos, UNALM, Av. La Molina s/n La Molina, Lima, Perú.

* Autor por correspondencia: liaseptiembre2012@hotmail.es; liarf@lamolina.edu.pe
} 
En los últimos diez años la producción nacional de arroz cáscara aumentó de 1962,000 toneladas a 2783,000 toneladas, lo que representa una tasa anual promedio del 4,2\%. El consumo de agua en el Perú, tanto en la costa como en la selva, está comprendido entre $12000 \mathrm{~m}^{3} \mathrm{ha}^{-1} \mathrm{y}$ $20000 \mathrm{~m}^{3}$ ha $^{-1}$ (Heros 2012).

La estimación del Kc con base en el clima local, la variedad y las condiciones de manejo del riego para el arroz ayudará a mejorar la programación del riego y la eficiencia del uso del agua, seguido de buenos rendimientos. Por ello, el uso del lisímetro aportará información útil para estimar la evapotranspiración del cultivo (ETc) y cuantificar el Kc a lo largo de todo el ciclo del cultivo, además de satisfacer las necesidades hídricas del arroz, racionalizando su uso y su disponibilidad hídrica en el suelo.

El cambio climático, el acelerado crecimiento poblacional y la alta demanda de agua en la agricultura han motivado esta investigación. El objetivo se centró en cuantificar el coeficiente del cultivo $(\mathrm{Kc})$ del arroz empleando un lisímetro de drenaje. Además, se buscó determinar la evapotranspiración de referencia (ETo) mediante el método de FAO Penman-Monteith y la evapotranspiración del cultivo (ETc) a partir de las medidas de un lisímetro de drenaje.

\section{Materiales y métodos}

El experimento se realizó del 20 de diciembre del 2017 al 30 de abril del 2018 en el Área Experimental de Riego (AER) de la Universidad Nacional Agraria La Molina (UNALM), situada en las coordenadas latitud sur $12^{\circ} 4$ '41,77'S, longitud oeste $76^{\circ} 56^{\prime} 45.20$ ' O y a una altitud de $248 \mathrm{msnm}$. en la zona geográfica 18L. El AER cuenta con una instalación para aplicar el sistema de riego presurizado, un reservorio de $15 \mathrm{~m}^{3}$, un cabezal de riego y un sistema de fertirriego.

\section{Material vegetal}

Se utilizó semilla de arroz variedad IR 71706

\section{Estación meteorológica automática}

(Davis Modelo Vantage Pro 2 Consola pantalla LCD 90 x $150 \mathrm{~mm}$ ): permitió obtener los datos meteorológicos necesarios para estimar los valores de ETo.

\section{Dos sensores GS1 del tipo FDR}

(Reflectometría en el dominio de la frecuencia) y un registrador de datos de la serie Decágon EM50 permitieron obtener datos de humedad volumétrica del suelo.

\section{Características físicas y químicas del suelo}

La caracterización físicoquímica se obtuvo al enviar muestras representativas de suelo al Laboratorio de Análisis de Agua, Suelo, Medio Ambiente y Fertirriego de la Facultad de Ingeniería Agrícola de la UNALM. Las muestras fueron obtenidas de diferentes puntos del área experimental, a una profundidad de 0 a $30 \mathrm{~cm}$. De acuerdo al análisis, el suelo presenta una textura franca arenosa y una densidad aparente de $1,54 \mathrm{~g} \mathrm{~cm}^{-3}$, la cual se determinó mediante el método del cilindro. Al analizar las características químicas del suelo, se clasificó como muy ligeramente salino (CE menor a $2 \mathrm{dS} / \mathrm{m})$, moderadamente alcalino $(\mathrm{pH}$ entre 7,9-8,4), presentó un nivel medio de materia orgánica (entre 2 y $4 \%$ ), se identificó alta presencia de fósforo (P menor a $70 \mathrm{ppm}$ ) y un contenido alto de potasio (P mayor a 240 ppm). Es importante mencionar que, antes de la siembra, se efectuaron riegos profundos para lavar las sales.

\section{Agua}

La fuente de agua proviene del río Rímac a través del canal de Ate y se deriva hacia el AER mediante canales revestidos, para ser almacenada en un reservorio de $15 \mathrm{~m}^{3}$. De acuerdo al análisis de laboratorio, el pH del agua fue de 7,4, por lo que es considerada neutra. La salinidad fue de $0,63 \mathrm{dS} / \mathrm{m}, \mathrm{y}$ pertenece a la clasificación C2. Es decir, la salinidad en el agua fue moderada. La relación de adsorción de sodio (RAS) fue de 0,56 bajo la clasificación S1, la cual indica que el nivel de sodio no representa peligro alguno. Por consiguiente, al considerar la salinidad y la presencia de sodio en el agua, deducimos que la investigación se desarrolló con agua C2 - S1, lo que significa agua de buena calidad para cultivos.

\section{Instalación del lisímetro}

Para su diseño se contempló que los materiales sean económicos, pero sin dejar de considerar las exigencias técnicas que el dispositivo implica. 
La construcción del lisímetro consistió en adecuar un contenedor HDPE cuadrado (1m x 1m x 1m). Luego se diseñó un sistema colector mediante la unión de segmentos de tubería PVC perforado y empotrado en la superficie inferior del contenedor apoyado sobre una base de cemento con pendiente del $5 \%$. La zona de filtrado (B) estuvo compuesta por grava, confitillo, arena gruesa y fina, tal como se muestra en la Figura 1 (margen derecha). Además, se incorporó suelo cultivable a la zona de crecimiento radicular (A). También se instaló un tubo receptor del agua drenada hacia un depósito para su almacenamiento y posterior medición volumétrica.

El lisímetro contó con dos líneas de riego separadas cada $50 \mathrm{~cm}$ y laterales de riego de $16 \mathrm{~mm}$. Los goteros tuvieron un caudal de 3,75 1 $\mathrm{h}^{-1} \mathrm{y}$ un distanciamiento de $25 \mathrm{~cm}$. Finalmente, se instalaron dos sensores de humedad GS1 a 12 y $24 \mathrm{~cm}$ de profundidad (Figura 1).

\section{Estimación de la evapotranspiración de referencia ETo}

La investigación se realizó con datos meteorológicos de la estación automática Davis. Para estos fines, se obtuvo información meteorológica de los meses de diciembre del 2017 y de enero, febrero, marzo y abril del 2018. Los valores diarios de ETo fueron calculados por medio del método FAO Penman-Monteith.

\section{Estimación de la evapotranspiración del cultivo ETc}

La ETc fue la incógnita del balance hídrico del lisímetro drenaje. Se efectuó del siguiente modo: primero, la lámina de riego (R), la lámina drenada (D) y la variación de humedad del suelo $(\Delta \mathrm{SW})$ fueron evaluadas en el campo entre cada evento de riego, así como la precipitación (P). Luego, se utilizó la siguiente ecuación: $\mathrm{ETc}=\mathrm{R}$ $+\mathrm{P}-\mathrm{D} \pm \Delta \mathrm{SW}$.

\section{Determinación del coeficiente de cultivo (Kc)}

El coeficiente del cultivo $(\mathrm{Kc})$ es básicamente el cociente entre la evapotranspiración del cultivo (ETc) y la evapotranspiración del cultivo de referencia (ETo). Se procedió a obtener el valor del $\mathrm{Kc}$ mediante la siguiente ecuación: $\mathrm{kc}=\mathrm{ETc} \mathrm{ETo}^{-1}$

\section{Resultados y discusión}

\section{Evapotranspiración de referencia (ETo)}

De todos los datos meteorológicos monitoreados, se debe resaltar que el mes de febrero registró la mayor temperatura promedio: $25.8^{\circ} \mathrm{C}$. En la Figura 2 se presentan los registros meteorológicos durante la investigación.

Las precipitaciones fueron esporádicas y mínimas, registrándose valores mensuales entre 0.4 y $1.8 \mathrm{~mm}$, los cuales no se consideran significativos en el balance hídrico diario. Finalmente, la humedad relativa fluctuó entre 70 y $89 \%$, la velocidad del viento fue de 0,5 a $0,7 \mathrm{~m} \mathrm{~s}^{-1}$ y la radiación solar aumentó notoriamente por el cambio estacional en los meses de enero, febrero y marzo.

En la Figura 3 se puede observar que la ETo fue variando en función del cambio estacional. El valor máximo diario se presentó en los meses de enero y marzo con 5,2 y $5 \mathrm{~mm} \mathrm{día}^{-1}$ y el mínimo en diciembre
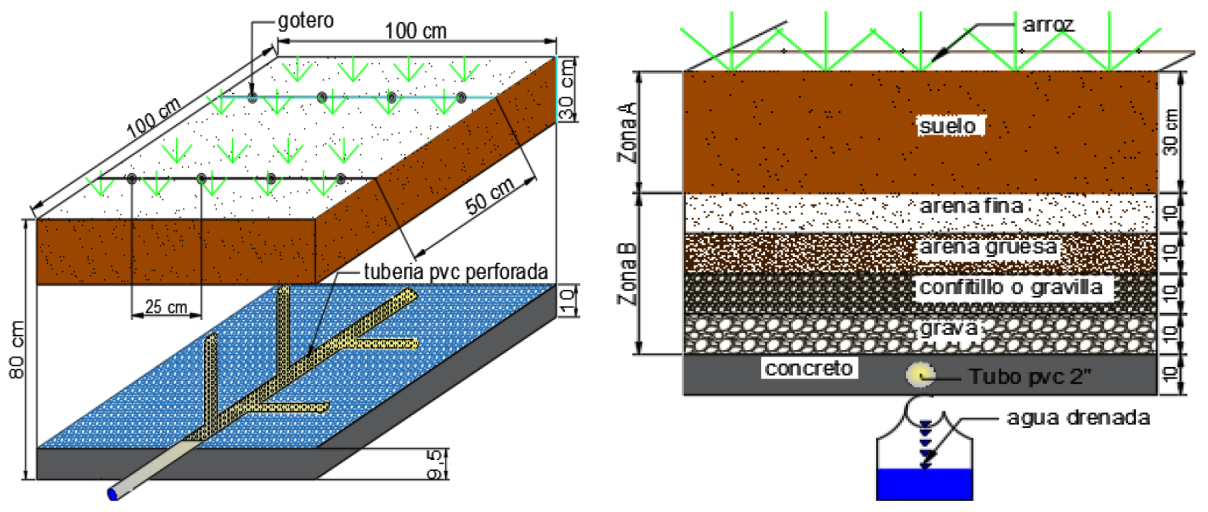

Figura 1. Lisímetro de drenaje: vista en 3D (panel izquierdo) y perfil (panel derecho). 

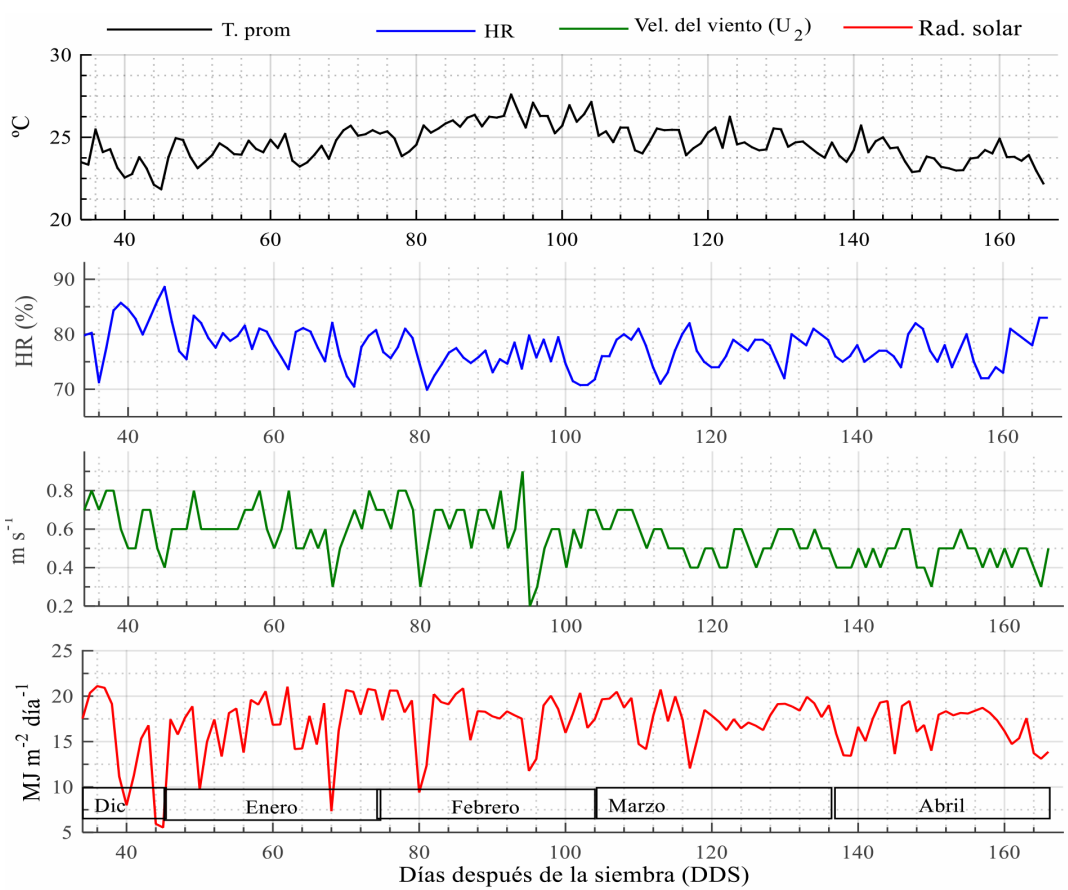

Figura 2. Variación temporal de la temperatura, humedad relativa, velocidad del viento y radiación Solar del 20/12/17 al 30/4/18.

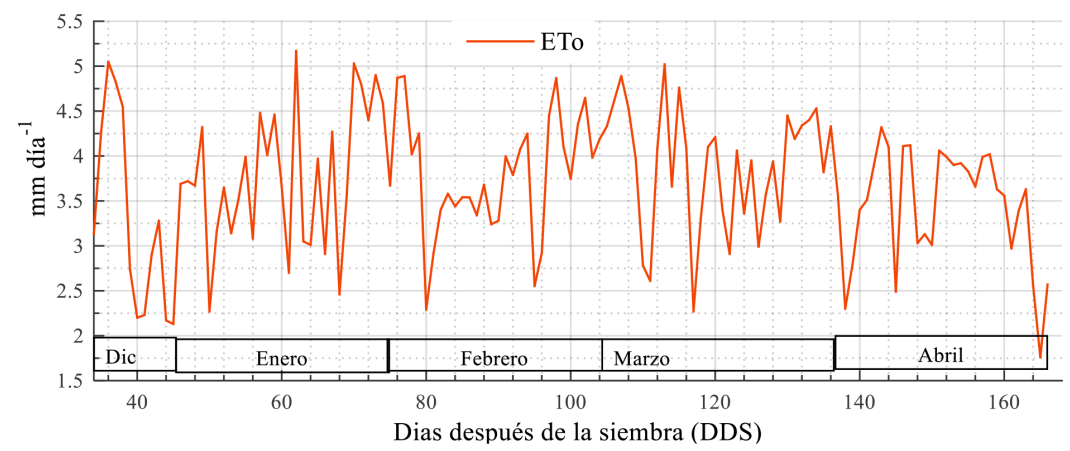

Figura 3. Variación temporal de la ETo del 20/12/17 al 30/4/18.

y abril con 2,1 y 1,8 mm día ${ }^{-1}$. Los meses con mayor ETo promedio fueron enero, marzo y febrero, y los más bajos diciembre y abril. La ETo total durante la investigación desde el 20 de diciembre del 2017 hasta el 29 de abril del 2018 fue de 481,88 mm, lo que es equivalente a $4.818,8 \mathrm{~m}^{3} \mathrm{ha}^{-1}$.

\section{Evapotranspiración del cultivo de arroz (ETc) IR 71706}

Para efectuar el balance de humedad del suelo, se evaluó la lámina de riego $(\mathrm{mm})$, la lámina drenada (mm), la variación de la humedad del suelo $(\mathrm{mm})$ y la precipitación $(\mathrm{mm})$. Además, se determinó el número de macollos y la altura de planta una vez por semana.

El suministro del riego se basó en mantener la humedad del suelo a capacidad de campo y reponer la lámina consumida del día anterior. En la fase vegetativa y reproductiva, la humedad varió entre $32 \%$ y $41 \%$. En la fase final, se midió entre $25-32 \%$ de humedad volumétrica (Figura 4).

En las Figuras 3 y 5 se muestra que la ETc está relacionada con el comportamiento de la ETo. 

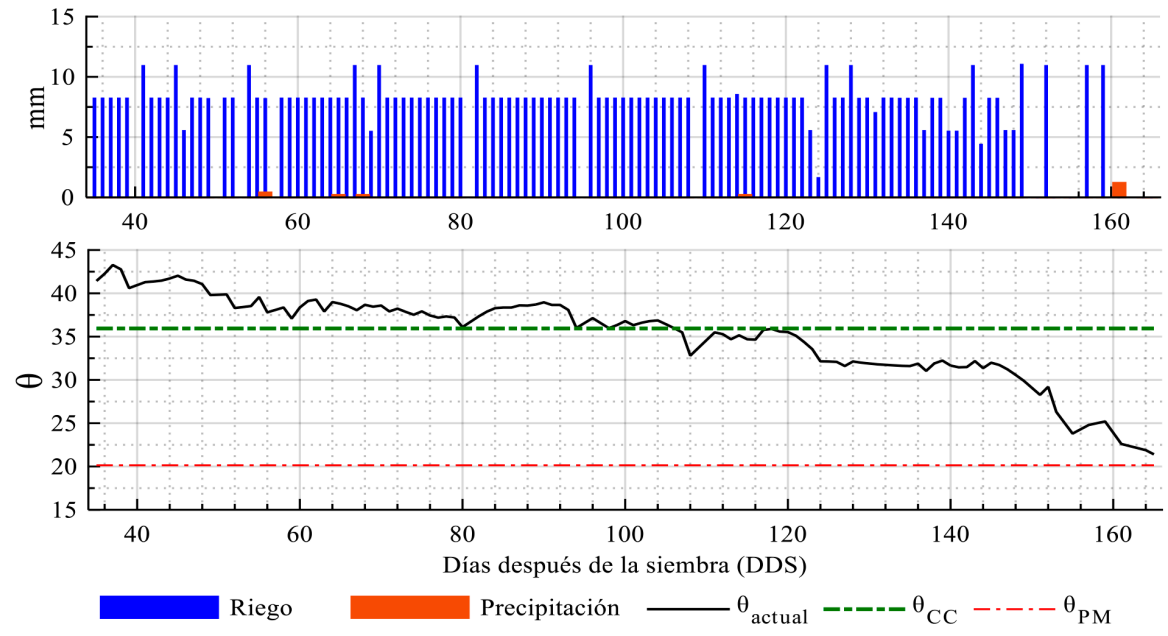

Figura 4. Variación temporal de la lámina de riego, precipitación y humedad volumétrica del suelo.

Por lo tanto, las tendencias de ETc y Transpiración siguen un mismo patrón de variación influenciado por la ETo, las características propias del cultivo y su estado fenológico. En la Figura 5 se puede observar la variación temporal de la ETc frente a la transpiración y evaporación. Es importante mencionar que el valor de transpiración se obtuvo en macetas evitando la evaporación directa del suelo.

\section{Coeficiente de cultivo (Kc) del arroz IR 71706}

Se presenta el Kc obtenido por etapa fenológica en el que se aprecia que los valores de Kc aumentan acorde al crecimiento del cultivo hasta la fase reproductiva (floración-antesis). Según Allent et al. (2006), la variación del Kc depende del área foliar (número de macollos), lo que implica un mayor número de estomas. También depende de la edad y altura de planta, y de la evaporación. Posteriormente, el Kc final declina por la senescencia propia del cultivo (Figura 6).

\section{Kc del arroz IR 71706 según las etapas definidas por la FAO}

La FAO presenta cuatro etapas de crecimiento del cultivo: inicial, desarrollo, medio y final, con tres valores de coeficiente único (promedio temporal) Kc- inicial, Kc-medio y Kc-final (Allent et al., 2006). En la Figura 7 se observa que los valores de Kc recomendados por la FAO para el cultivo de arroz están subestimados. Mohan y

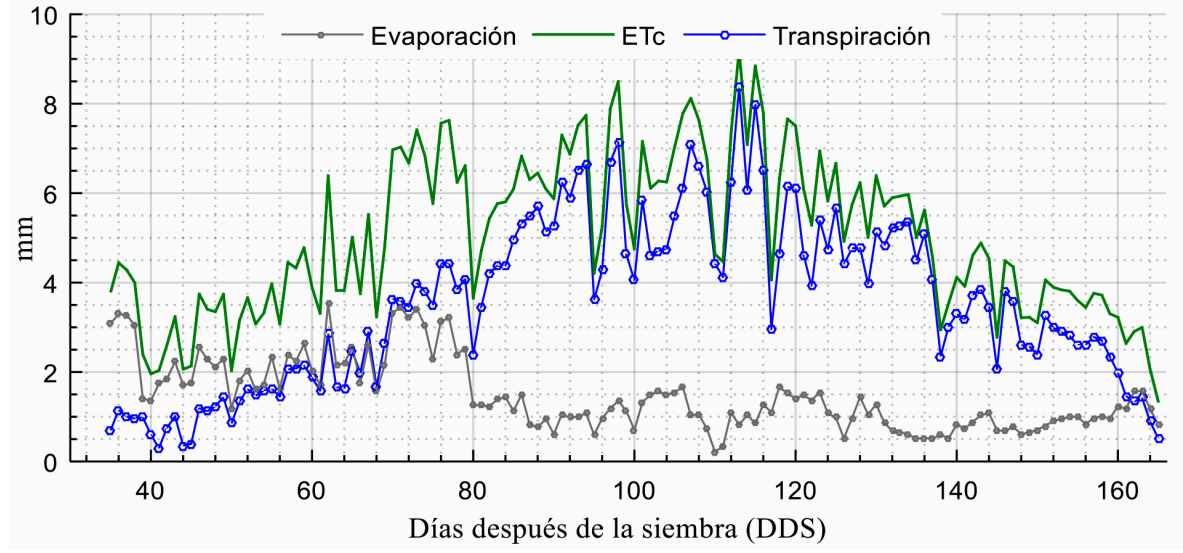

Figura 5. Variación temporal de la ETc y transpiración. 


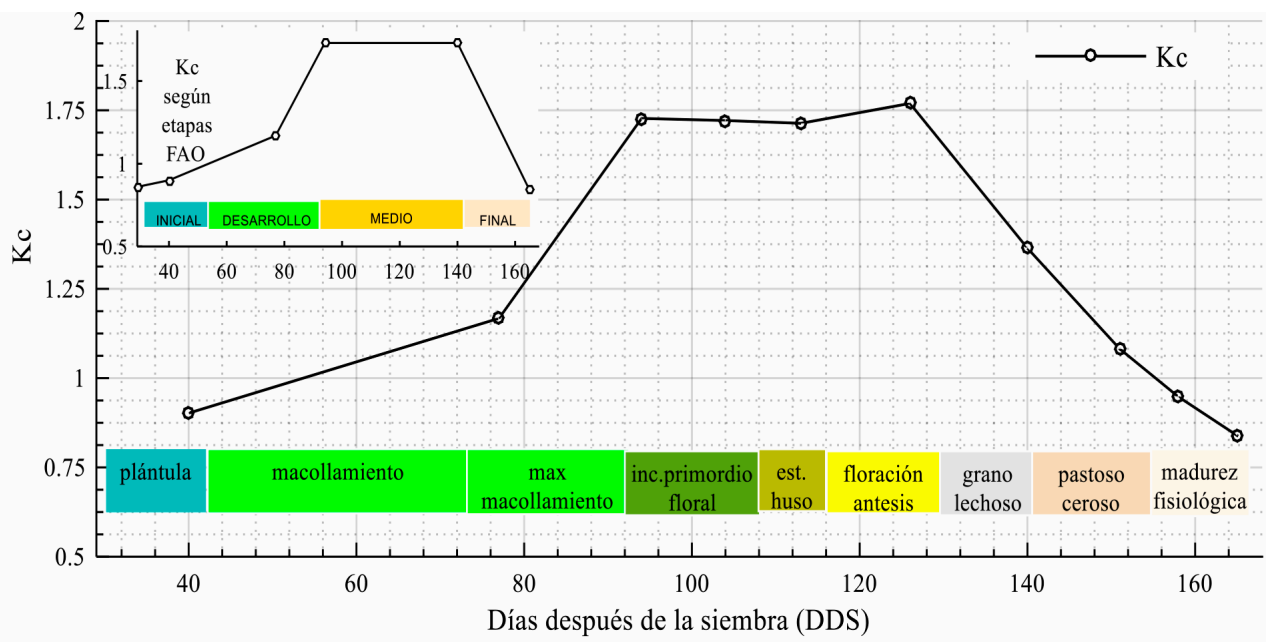

Figura 6. Variación temporal del Kc por etapa fenológica.

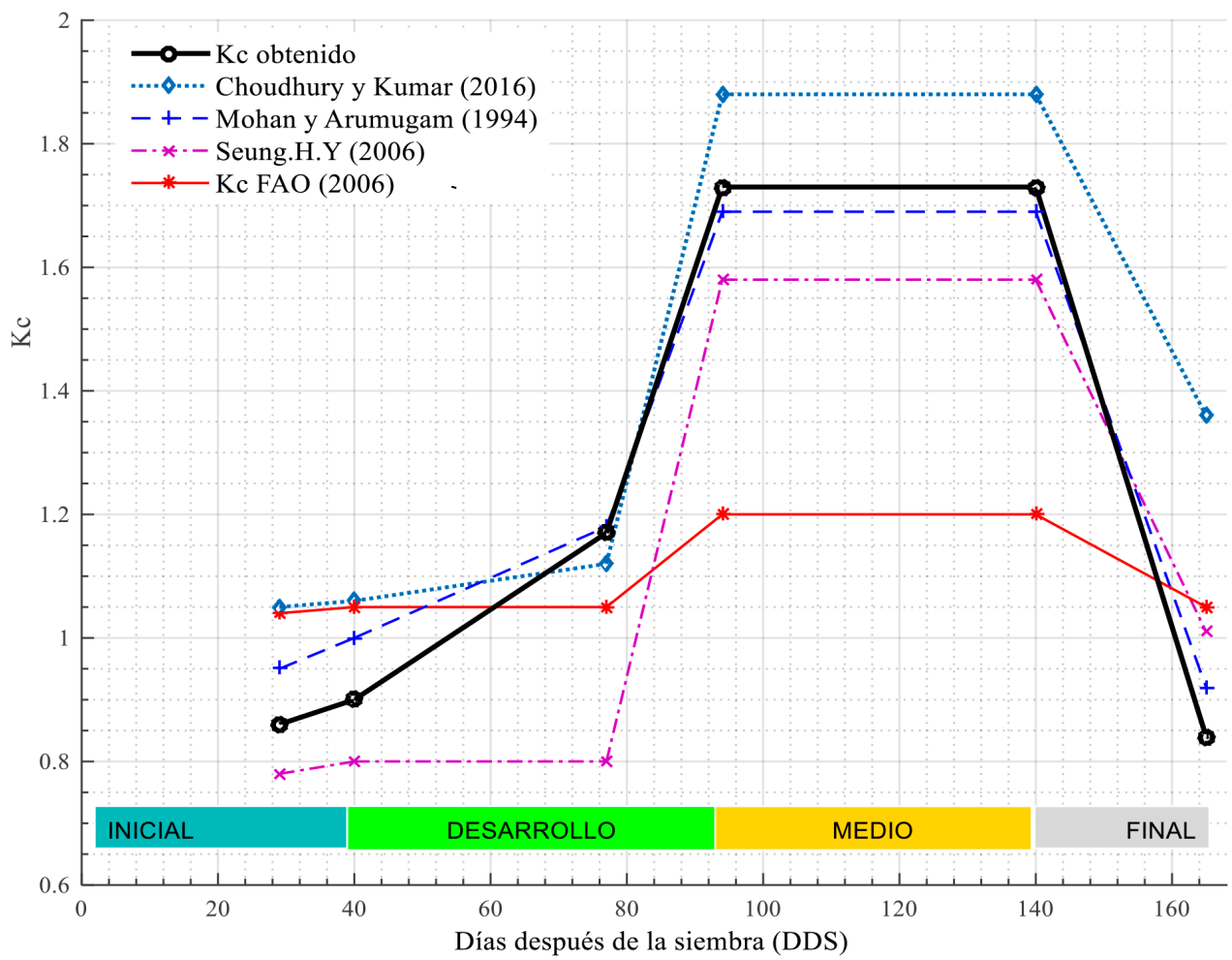

Figura 7. Kc obtenido comparado con el Kc de la FAO y otros.

Arumugam (1994) reportan valores de Kc en el rango de 0,95-1,69 que guardan estrecha relación con el Kc obtenido en el rango de 0,9 a 1,7. Por otro lado, Seung (2006) registra valores de Kc menores, y Choudhry y Kumar (2016) valores superiores al Kc obtenido.

\section{Eficiencia del uso del agua}

La eficiencia del uso de agua aplicada fue de $0,82 \mathrm{~kg}$ de grano por $\mathrm{m}^{3} \mathrm{y}$ un consumo específico del agua aplicada de $1.219,5 \mathrm{~m}^{3}$ por tonelada de grano. $\mathrm{La}$ productividad del agua consumida como ETc fue de 
$1,14 \mathrm{~kg}$ de grano por $\mathrm{m}^{3} \mathrm{y}$ un consumo específico de agua de $877,2 \mathrm{~m}^{3}$ por tonelada de grano cosechado. Gonzáles et al. (2015), utilizando riego por aspersión, reportan una eficiencia del uso de agua aplicada de $0.8 \mathrm{~kg}$ de arroz por $\mathrm{m}^{3} \mathrm{y}$ un consumo específico de $1250 \mathrm{~m}^{3}$ por tonelada de arroz cosechado. Jacob (2003) registra valores en el rango de 0,5 a $1,1 \mathrm{~kg} / \mathrm{m}^{3}$ sobre la productividad del agua como ETc en arroz.

\section{Conclusiones}

Los coeficientes del cultivo del arroz IR 71706 según las etapas definidas por la FAO (Kc inicial, Kc medio y Kc final) fueron de 0,$9 ; 1,70$ y 0,84 , respectivamente, bajo riego por goteo.

La evapotranspiración de referencia (ETo) obtenida por el método FAO Penman-Monteith varió de 1,75 a $5,16 \mathrm{~mm}^{\prime}$ dí$^{-1}$ con valor promedio de $3,78 \mathrm{~mm} \mathrm{día}^{-1}$ y $481,88 \mathrm{~mm}$ de ETo para todo el período vegetativo.

Del balance de humedad del suelo, se obtuvo la ETc, cuyo valor diario máximo fue de $9,19 \mathrm{~mm}$ día ${ }^{-1}$ en la etapa de estado de huso y floración y un valor mínimo de $1,35 \mathrm{~mm} \mathrm{día}^{-1}$ en la etapa final del cultivo, para un promedio de $5,02 \mathrm{~mm} \mathrm{día}^{-1}$. La ETc total fue de $658,23 \mathrm{~mm}$ para 587 macollos $\mathrm{m}^{-2}$ y una altura promedio de $80 \mathrm{~cm}$.

La eficiencia de uso del agua aplicada fue de $0,82 \mathrm{~kg}$ de grano por $\mathrm{m}^{3}$ y un consumo específico del agua aplicada de $1219,5 \mathrm{~m}^{3}$ por tonelada de grano. La productividad del agua consumida como ETc fue de $1,14 \mathrm{~kg}$ de grano por $\mathrm{m}^{3} \mathrm{y}$ un consumo específico de agua de $877,2 \mathrm{~m}^{3}$ por tonelada de grano.

\section{Literatura citada}

Allen, R; Pereira, L; Raes, D; Smith, M.

2006. Evapotranspiración del cultivo: Guías para la determinación de los requerimientos de agua de los cultivos. Organización de las Naciones Unidas para la Agricultura y la Alimentación (FAO). Roma, Italia. 298 p.

Choudhury, B.U; Kumar, A.S.

2016. Estimation of crop coefficient of irrigated transplanted puddled rice byfield scale water balance in the semi-arid Indo-Gangetic Plains, India. Revista Agricultural Water Management., 176: 142-150.

Gonzales, R; López, S; Herrera, P.

2015. Indicadores de la productividad del agua por cultivos y técnicas de riego en Cuba. Revista Ciencias Técnicas Agropecuarias, 24(4): 57-63.

Heros, E.

2012. Guía técnica: Manejo técnico del manejo integrado del arroz. UNALM. Lima, Perú. 45 p.
Mohan, S; Arumugam, N.

1994. lrrigation crop coefficients for lowland rice. Revista Kluwer Academic Publishers. Printed in the Netherlands, 8: $159-176$.

Jacob, W.

2003. Descubrir el potencial del agua para la agricultura. Organización de las Naciones Unidas para la Agricultura y la Alimentación (FAO). Roma, Italia. 62 p.

Seung, $\mathrm{H}$.

2006. Estimation of Paddy Rice Crop Coefficients for Penman-Monteith and FAO Modified Penman Method. Revista ASABE-American Society of Agricultural and Biological Engineers, DOI: 10.5389/KSAE.2006.48.1.013. Tabra, S.

2013. La preocupante y desigual situación del agua en el Perú. SERVINDI. Boletín 201. 6 p. 
